\title{
Microsphere enabled subdiffraction- limited optical-resolution photoacoustic microscopy: a simulation study
}

\author{
Paul Kumar Upputuri \\ Moganasundari Krisnan \\ Manojit Pramanik
}




\title{
Microsphere enabled subdiffraction-limited optical-resolution photoacoustic microscopy: a simulation study
}

Paul Kumar Upputuri, Moganasundari Krisnan, and Manojit Pramanik

Nanyang Technological University, School of Chemical and Biomedical Engineering, 62 Nanyang Drive, Singapore 637459, Singapore

\begin{abstract}
Optical resolution photoacoustic microscopy (ORPAM) is a high-resolution hybrid imaging modality having potential for microscale in vivo imaging. Optical diffraction limits the lateral resolution of ORPAM. A photonic nanojet (PNJ) was used to break this diffraction limit. A single round microsphere can generate a PNJ with subwavelength waist, but its short axial length limits its applications to surface imaging only. We investigate different sphere designs to achieve ultralong nanojets that will make the nanojet more viable in far-field applications, such as photoacoustic imaging. The PNJ properties, including effective length, waist size, working distance, and peak intensity, can be tuned and controlled by changing the sphere design and its refractive index. A truncated multilayer microsphere design could generate an ultraelongated PNJ with length larger than $\sim 172 \lambda(\sim 138 \mu \mathrm{m})$ while retaining a large working distance $\sim 32 \lambda(\sim 26 \mu \mathrm{m})$. Through simulation study, we observed $\sim 11$-fold enhancement in lateral resolution with $5 \mu \mathrm{m}$ round sphere (refractive index 2.2) when used in a conventional ORPAM setup with NA $=0.1$ and $\lambda=800 \mathrm{~nm}$. $\odot 2016$ Society of Photo-Optical Instrumentation Engineers (SPIE) [DOI: 10.1117/1.JBO.22.4.045001]
\end{abstract}

Keywords: photoacoustics; microsphere; photonic nanojet; subdiffraction; super-resolution; micro-optics.

Paper 160554SSRR received Aug. 15, 2016; accepted for publication Nov. 7, 2016; published online Nov. 30, 2016.

\section{Introduction}

Photoacoustic microscopy (PAM) is a hybrid in vivo threedimensional (3-D) imaging modality that can offer high-resolution images with optical absorption contrast. ${ }^{1}$ PAM has so far been applied to numerous applications such as vascular biology, oncology, neurology, ophthalmology, dermatology, gastroenterology, and cardiology. ${ }^{2-6}$ In PAM, short laser pulse energy is absorbed by an intrinsic absorber, such as hemoglobin or melanin in tissue, leading to local temperature rise, and due to thermoelastic expansion generates pressure waves in the form of ultrasound. The photoacoustic (PA) waves are detected with an ultrasonic transducer (UST). PAM has several advantages over pure optical methods: ${ }^{1}$ (a) PAM makes use of the weak acoustic scattering to do high-resolution imaging beyond the optical diffusion limit $(\sim 1 \mathrm{~mm})$ in biological tissues, (b) it is highly scalable 3-D in vivo imaging modality, both imaging depth and resolution are scalable, (c) it provides optical absorption contrast, unlike confocal fluorescence microscopy and optical coherence tomography (OCT), which provides fluorescence and scattering contrast, (d) it directly images optical absorption with $100 \%$ relative sensitivity, and the sensitivity is two orders of magnitude greater than those of confocal microscopy and $\mathrm{OCT}^{2}$ and (e) it can simultaneously image functional, structural, molecular, flow dynamic, and metabolic contrasts in vivo.,

In PAM, both the laser excitation and acoustic detection are focused (tightly or loosely depending on the configuration), and the dual foci are configured confocally to enhance measurement sensitivity. Depending on whether the acoustic focus or optical focus is finer, PAM is categorized into acoustic-resolution
PAM (ARPAM) ${ }^{8}$ and optical-resolution PAM (ORPAM). ${ }^{9-12}$ In ARPAM, the acoustic focus is finer, and it could provide an axial resolution of $15 \mu \mathrm{m}$, and a lateral resolution of $45 \mu \mathrm{m}$ at 3mm depth with $0.44 \mathrm{NA}, 50 \mathrm{MHz} \mathrm{UST}^{8}{ }^{8}$ In ORPAM, the optical focus is finer. So, the size of optical spot limits the imaging resolution in ORPAM. The diffraction-limited size of optical focus is $R=0.5 \lambda / \mathrm{NA}$, where $\lambda$ denotes the optical wavelength and $\mathrm{NA}$ is the numerical aperture of the optical objective. ${ }^{2}$ One way to reduce the spot size is to use high-NA objective. ORPAM systems with resolution $5 \mu \mathrm{m}$ using $0.1 \mathrm{NA}$ and $\lambda=590 \mathrm{~nm},{ }^{13} 2.9 \mu \mathrm{m}$ using $0.1 \mathrm{NA}$ and $\lambda=570 \mathrm{~nm}, 6.2 \mu \mathrm{m}$ using $0.1 \mathrm{NA}$ and $\lambda=1046 \mathrm{~nm},{ }^{14} 2 \mu \mathrm{m}$ using $0.47 \mathrm{NA}$ and $\lambda=532 \mathrm{~nm},{ }^{15}$ and $0.22 \mu \mathrm{m}$ using $1.23 \mathrm{NA}$ and $\lambda=532 \mathrm{~nm}^{12}$ were demonstrated. Because of the high sensitivity and resolution, ORPAM has become an important tool for studying biological systems at the cellular and subcellular levels. In vivo label-free PAM of cell nuclei by excitation of DNA and RNA was demonstrated using UV light excitation. ${ }^{16}$ In all these techniques, the resolution is still limited by the optical diffraction. It is necessary to achieve imaging resolution beyond the diffraction limit to image subcellular structures, mitochondria, hemoglobin nanoclusters, and nanoparticles. In recent years, few attempts have been made to break the diffraction limit in ORPAM. A nonlinear super-resolution PAM was demonstrated for visualizing 10-nm gold nanoparticles in graphene and hemoglobin nanoclusters in live erythrocytes. ${ }^{17} \mathrm{~A}$ label-free photoacoustic nanoscopy (PAN) using optical saturation and polynomial fitting was demonstrated for imaging mitochondria in NIH $3 \mathrm{~T} 3$ fibroblasts. ${ }^{18}$ Using a double-excitation process, 
subdiffraction-limited lateral resolution was demonstrated in photoimprint PAM. ${ }^{9}$ These approaches are either complicated or expensive or both.

Dielectric microspheres were widely used to break the diffraction limit in optical microscopy. ${ }^{19-22}$ It is simpler, cost-effective, and can provide far-field super-resolution. This microsphere approach can be implemented in conventional microscopy without modifying the system and it has gained enormous attention in the field of optical nanoscopy. ${ }^{21,23}$ Optical imaging at 50-nm resolution was achieved in white light microscopy using fused silica $\left(\mathrm{SiO}_{2}\right)$ microspheres with diameter in the range 4 to $9 \mu \mathrm{m}$ in air medium. ${ }^{21}$ The super-resolution is due to a photonic nanojet (PNJ) generated by the microsphere. When the microsphere is illuminated by a light beam, a narrow (subwavelength waist), intense, nonevanescent, and low-diverging jet like optical beam is generated on the shadow side of the microsphere. Such a nanojet can break the diffraction limit in optical microscopy by greatly reducing the size of the excitation volume, and the signal generated in the excitation volume can still be collected in the far-field. Super-resolution imaging using a PNJ was successfully demonstrated in white light microscopy, ${ }^{21}$ fluorescence microscopy, ${ }^{24}$ Raman microscopy, ${ }^{25}$ and coherent anti-stokes Raman scattering microscopy. ${ }^{26}$

A simulation study was reported on achieving super-resolution in ORPAM using PNJs. ${ }^{27}$ This study was focused on: (i) generation of PNJ by a round microsphere (RM) under tight optical focusing (using high-NA values $0.5,0.7$, and 1.0) condition in water, and (ii) one of the important PNJ parameters, its waist size, was studied by changing the incident wavelength $(\lambda)$, numerical aperture (NA) of the objective, refractive index of the microsphere $(n 1)$, and diameter of the sphere $(D)$. It was observed that RM could generate a narrow jet, but its major drawback is its short length which limits its penetration depth. To control PNJ properties, several designs such as truncated microsphere (TM), ${ }^{28}$ microsphere with concentric rings (MCR), ${ }^{29}$ and multilayer microsphere (MLM) ${ }^{30,31}$ have been reported till date.

In this work, we investigated all the important parameters of PNJs including "effective length" related to the penetration depth, "waist size" related to the lateral resolution, "working distance" related to the distance between the shadow-side surface of the sphere to the sample, and "peak intensity" related to the enhancement of the signal. We compared PNJ parameters for various sphere designs such as RM, TM, MCR, MLM, and a new design named truncated multilayer microsphere (TMLM). Here, an $800 \mathrm{~nm}$ Gaussian beam was loosely focused (low NA) on the sphere to generate PNJ. The low NA (0.1) used in this work is also in line with the widely used NA in ORPAM studies. ${ }^{13,14}$ The TMLM design generated a PNJ with remarkable length $\sim 138 \mu \mathrm{m}$ (i.e., $\sim 172 \lambda$, it is the longest nanojet achieved so far) and focal length $\sim 26 \mu \mathrm{m}$ (i.e., $\sim 32 \lambda$, it is the longest focal length achieved with microsphere so far). We also observed that using RM with $D \sim 5 \mu \mathrm{m}$ and $n 1=2.2$ it is possible to achieve $\sim 11$-fold enhancement in the lateral resolution in conventional ORPAM with $0.1 \mathrm{NA}, \lambda=800 \mathrm{~nm}$.

\section{Methods}

COMSOL multiphysics is a finite element method (FEM)-based simulation software package for various physics and engineering applications. COMSOL software version $4.3 \mathrm{~b}$ was used for generating the fluence maps for Gaussian focus and PNJs. These fluence maps were then exported to MATLAB for PA image simulation using the k-wave toolbox. k-wave $\mathrm{e}^{32}$ is an open source acoustics tool box in MATLAB. It was designed for ultrasound and PA simulations in tissue media. For both COMSOL and k-wave-based simulations, a desktop with 64-bit windows 10 operating system, $3.7 \mathrm{GHz}$ processor, and $16 \mathrm{~GB}$ RAM was used.

\subsection{COMSOL-Based Simulation for Generating Focused Gaussian Beam}

We designed a two-dimensional (2-D) FEM simulation geometry based on electromagnetic theory in COMSOL. The simulation model used is illustrated in Fig. 1(a). Area of simulation $(20 \mu \mathrm{m} \times 250 \mu \mathrm{m})$ includes optical focusing, water medium, and microsphere. Perfect matching layer (PML) was used as a boundary condition. A focused beam was simulated by a Gaussian beam propagating along the $+y$-axis with a full width at half maximum (FWHM) beam waist diameter of $\lambda / 2$ NA or an $e^{-2}$ radius of $\lambda / 2.35 \mathrm{NA}$ in intensity, ${ }^{26}$ where $\lambda$ is the incident wavelength and NA is the numerical aperture of the objective. The beam waist was always centered laterally in the simulation area $(x=0)$, but the $y$-position varies for different simulations. In our simulations, we have generated a focusing Gaussian beam with $\lambda=800 \mathrm{~nm}$ and NA $=0.1$, so its theoretical FWHM waist diameter would be $4000 \mathrm{~nm}$.

\subsection{Simulation of Photonic Nanojets in COMSOL}

The FEM model shown in Fig. 1(a) was used for generating PNJs. The dielectric solid microsphere with diameter $D$ and refractive index $n 1$ was immersed in water medium having refractive index $n 0=1.33$. In most of the previously reported PNJ simulations, the incident light was a plane wave. But in ORPAM, excitation beam is a focused Gaussian beam. Hence, in our simulations, we have used $800 \mathrm{~nm}$ Gaussian beam focused using an objective with $\mathrm{NA}=0.1$ as discussed in Sec. 2.1. A sphere with $D=5 \mu \mathrm{m}$ was placed inside the Gaussian focus having an FWHM diameter of $3958 \mathrm{~nm}$. Spheres with different designs and different materials were tested. The materials used for the spheres were all existing materials. We have carried out simulations for the following spheres: (i) RM: It was a perfectly round, solid sphere with a diameter, $D=5 \mu \mathrm{m}$. The material used for the sphere is $n 1=1.45$ (fused silica), $n 1=1.58$ (polystyrene), or $n 1=2.2\left(\mathrm{TiO}_{2}-\mathrm{BaO}-\mathrm{ZnO}\right.$ glass). This design was widely used for generating PNJs. ${ }^{21,27}$ (ii) TM: Through simulation studies, it was observed that PNJ parameters are significantly influenced just by truncating the RM. ${ }^{28}$ In our simulations, a $D=5-\mu \mathrm{m}$ diameter $\mathrm{RM}$ made out of silica was cut by different thicknesses, $T=0.25 D$, $0.5 D, 0.75 D$. So, the cutting thicknesses used here are $T=1.25,2.5,3.75 \mu \mathrm{m}$. (iii) MCR: Both experimental and simulation studies showed that the MCR on its shadow-side surface could modulate the PNJ generated. ${ }^{29}$ In our simulations, we have used a $D=5 \mu \mathrm{m}$ silica sphere decorated with 2,3 , or 4 uniformly distributed rings. The depth and width of all the rings were 1.2 and $0.25 \mu \mathrm{m}$, respectively. The inner radius of the first ring was $0.5 \mu \mathrm{m}$ and the distance between the adjacent rings was $0.25 \mu \mathrm{m}$. (iv) MLM: It was shown that using a two-layer microsphere configuration it is possible to enhance the length of the nanojet significantly. A PNJ with FWHM length of $\sim 22 \lambda(\sim 14 \mu \mathrm{m}),{ }^{31}$ and a PNJ with decay length of $\sim 100 \lambda(\sim 40 \mu \mathrm{m})^{30}$ were achieved using a two-layer microsphere. In our simulations, we have used an MLM sphere consisting of an inner core of radius $(r 1)$ and with a refractive index 


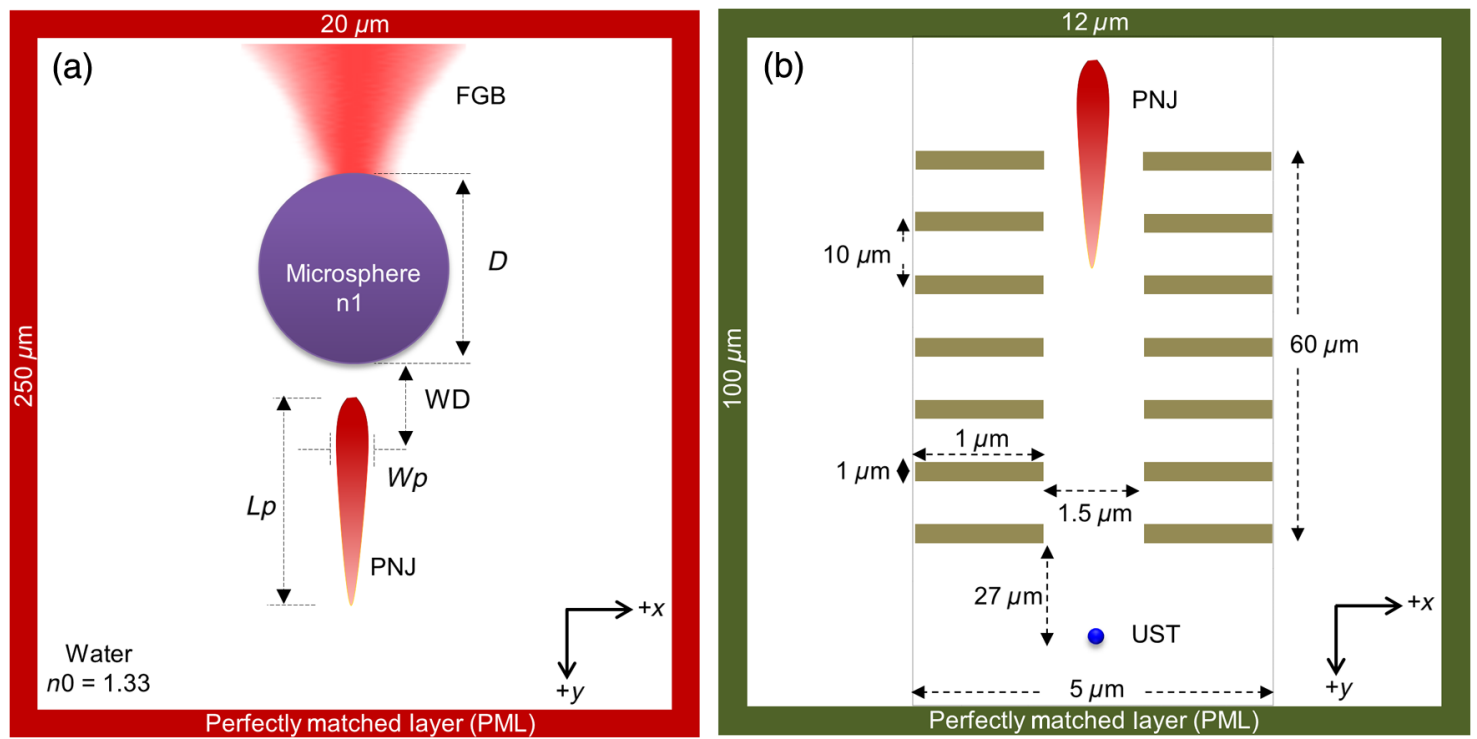

Fig. 1 (a) Simulation geometry for PNJ generation using FEM in COMSOL: FGB-focused Gaussian beam, $n 0$-refractive index of water, $n 1$-refractive index of microsphere, $D$-diameter of microsphere, WD-working distance, $W_{p}-\mathrm{FWHM}$, and $L_{p}$-effective length of PNJ. (b) Simulation geometry for PA microscopic imaging using k-wave in MATLAB. UST—ultrasound transducer (point detector).

$(n 1)$, and of a shell with outer radius $(r 2)$ and with an index $(r 2)$. The schematic of microspheres is shown in Fig. 2. The total diameter of the two-layer sphere was $D=5 \mu \mathrm{m}$. Three different two-layered spheres were designed with existing material: (a) silica sphere $(n 1=1.45)$ and $\mathrm{BK} 7$ layer $(n 2=1.51)$, (b) BK7 sphere $(n 1=1.51)$ and $\mathrm{MgF}_{2}$ layer $(n 2=1.38)$, (c) lanthanum dense flint (LaSF) sphere $(n 1=1.83)$, and barium flint $(\mathrm{BaF})$ layer $(n 2=1.60)$. (v) TMLM: The twolayer spheres discussed above were truncated to study the influence of its cutting thickness on the PNJ parameters. We found that truncating the MLM design significantly enhanced the effective length, and working distance compared to all other designs. The three different spheres used for MLM design were truncated by thickness, $T=0.25 D, 0.5 D, 0.75 D$ (i.e., $T=1.25,2.5,3.75 \mu \mathrm{m})$. Figure 2 summarizes the properties of PNJs generated from the five different designs discussed above. Figure 2 is discussed in detail in Sec. 3.

\subsection{Simulation of Photoacoustic B-Scan Images Using k-Wave Toolbox in MATLAB}

The fluence maps of focusing Gaussian beam and PNJs were exported to MATLAB for simulating PA images using the kwave toolbox. Figure 1(b) shows the 2-D simulation geometry used in k-wave simulations. A grid of $251 \times 2001$ pixels (i.e., $12 \mu \mathrm{m} \times 100 \mu \mathrm{m}$ ) was created with $50 \mathrm{~nm} /$ pixel. A PML was used as a boundary condition for the generation of the forward PA data. A numerical phantom as shown in Fig. 1(b) was used for the study. The phantom consists of square targets with a side length of $1 \mu \mathrm{m}$, separated by a distance $1.5 \mu \mathrm{m}$. Seven such targets were placed along $+y$-axis with $10 \mu \mathrm{m}$ gap between each pair of targets to create an object with a depth of $60 \mu \mathrm{m}$. The targets were embedded inside a nonabsorbing medium.

In our simulation, the light illumination was fixed at the center of the scanning area and the object was scanned across the light field. The scanned area was $101 \times 2001$ pixels (i.e., $5 \mu \mathrm{m} \times 100 \mu \mathrm{m})$. The object was moved along the $x$-direction with a step size of $50 \mathrm{~nm}$ resulting in a total of 101 A-lines. A point detector (UST) was used to collect the A-lines and it was placed at a distance of $27 \mu \mathrm{m}$ from the object. The center frequency and the fractional bandwidth of the detector were 375 or $1225 \mathrm{MHz}$ and $80 \%$, respectively. According to the simulations, 375 and $1225 \mathrm{MHz}$ transducers can provide up to $\sim 1$ - and $\sim 0.1-\mathrm{mm}$ depths, respectively, at $-20 \mathrm{~dB}$ signal attenuation. ${ }^{33-35}$ To simplify the simulations, we used a point detector. To collect forward PA signal over a length of $100 \mu \mathrm{m}$, a total of 1340 time steps with each step $50 \mathrm{ps}$ was used. The PA signals were down sampled five times to mimic a data acquisition system with $4 \mathrm{GS} / \mathrm{s}$ sampling rate. To every PA signal, $1 \%$ noise was added, resulting in $40 \mathrm{~dB}$ signal-to-noise ratio level. The A-line PA data were stacked after taking Hilbert transform to form a B-scan PAM image. The simulations assumed a sound speed of $1500 \mathrm{~m} / \mathrm{s}$. For simplicity, the medium was considered acoustically homogeneous and there was no absorption or dispersion of sound.

\section{Results and Discussion}

\subsection{Properties of Photonic Nanojets from Various Sphere Designs}

As discussed in Sec. 2.1, a Gaussian focus was generated with $\mathrm{NA}=0.1$ and $\lambda=800 \mathrm{~nm}$, and its FWHM waist diameter measured to be $W_{g}=3958 \mathrm{~nm}$. As discussed in Sec. 2.2, the simulations were carried out to investigate PNJs for sphere designs RM, TM, MCR, MLM, and TMLM. The basic characteristics of PNJ are as follows: (i) effective length $\left(L_{p}\right)$ is the distance of the point where the nanojet intensity drops by a factor of $(1 / e)$ with respect to the peak intensity $\left(I_{p}\right)$ in the axial profile ( $+y$-axis), (ii) working distance (WD) is the distance from the sphere shadow-side surface to the point of peak intensity $\left(I_{p}\right)$, (iii) waist of the nanojet $\left(W_{p}\right)$ is the FWHM of the nanojet intensity profile (along $+x$-axis) at peak intensity $\left(I_{p}\right)$, (iv) peak intensity $\left(I_{p}\right)$ is the maximum intensity of the PNJ, (v) figure-of-merit or quality criterion " $Q$ " is a measure of the quality of the PNJ, it combines the basic characteristics of PNJ, and is defined as $Q=\left(L_{p} \times I_{p}\right) / W_{p} \cdot{ }^{36}$ For a given microsphere, the PNJ was 
Upputuri, Krisnan, and Pramanik: Microsphere enabled subdiffraction-limited optical-resolution...

\begin{tabular}{|c|c|c|c|c|c|c|c|c|}
\hline \multirow{2}{*}{$\begin{array}{l}\text { Design } \\
\text { Code }\end{array}$} & \multirow{2}{*}{$\begin{array}{l}\text { Design of the } \\
\text { Sphere }\end{array}$} & \multirow{2}{*}{\multicolumn{2}{|c|}{$\begin{array}{l}\text { Optical and Geometric } \\
\text { Parameters of the Sphere }\end{array}$}} & \multicolumn{5}{|c|}{ PNJ Parameters } \\
\hline & & & & $\mathrm{Lp}(\mathrm{nm})$ & $\mathrm{Wp}(\mathrm{nm})$ & WD (nm) & $\operatorname{Ip}\left(v^{2} / \mathrm{m}^{2}\right)$ & $\mathrm{Q}=(\mathrm{Lp} * \mathrm{Ip}) / \mathrm{Wp}$ \\
\hline \multirow{3}{*}{$\mathrm{RM}$} & \multirow{3}{*}{$\mathrm{n} 1$} & \multicolumn{2}{|c|}{$\mathrm{n} 1=1.45$} & $11779^{*}$ & 909 & 5954 & 2.56 & 33.2 \\
\hline & & \multicolumn{2}{|c|}{$\mathrm{n} 1=1.58$} & 4639 & 563 & 2535 & 3.54 & 29.2 \\
\hline & & \multicolumn{2}{|c|}{$\mathrm{n} 1=2.20$} & 1380 & 360 & 295 & 4.87 & 18.7 \\
\hline \multirow{3}{*}{$\mathrm{TM}$} & & \multirow{3}{*}{$\mathrm{n} 1=1.45$} & $\begin{array}{l}\mathrm{T}=0.25 \mathrm{D} \\
=1.25 \mu \mathrm{m}\end{array}$ & 17559 & 1079 & 9167 & 2.18 & 35.5 \\
\hline & & & $\begin{array}{l}\mathrm{T}=0.50 \mathrm{D} \\
=2.50 \mu \mathrm{m}\end{array}$ & 49936 & 1339 & 11479 & 1.44 & 53.7 \\
\hline & & & $\begin{array}{l}\mathrm{T}=0.75 \mathrm{D} \\
=3.75 \mu \mathrm{m}\end{array}$ & 74314 & 1854 & 11266 & 1.23 & 49.3 \\
\hline \multirow{3}{*}{ MCR } & \multirow{3}{*}{$\mathrm{nl} \quad 0.25 \mu \mathrm{m}$} & \multirow{3}{*}{$\mathrm{n} 1=1.45$} & $\mathrm{~N}=2$ & 11779 & 863 & 5958 & 2.30 & 31.4 \\
\hline & & & $\mathrm{N}=3$ & 14019 & 913 & 5754 & 2.35 & 36.1 \\
\hline & & & $\mathrm{N}=4$ & 12959 & 903 & 5856 & 2.48 & 35.6 \\
\hline \multirow{3}{*}{ MLM } & & $\begin{aligned} \mathrm{r} 1 & =2.4 \mu \mathrm{m} \\
\mathrm{r} 2 & =2.5 \mu \mathrm{m}\end{aligned}$ & $\begin{array}{l}\mathrm{n} 1=1.45 \\
\mathrm{n} 2=1.51\end{array}$ & 4640 & 603 & 3360 & 3.07 & 23.6 \\
\hline & & $\begin{aligned} \mathrm{r} 1 & =2.4 \mu \mathrm{m} \\
\mathrm{r} 2 & =2.5 \mu \mathrm{m}\end{aligned}$ & $\begin{aligned} \mathrm{n} 1 & =1.51 \\
\mathrm{n} 2 & =1.38\end{aligned}$ & 46896 & 1538 & 13719 & 1.43 & 43.6 \\
\hline & & $\begin{aligned} \mathrm{r} 1 & =2.0 \mu \mathrm{m} \\
\mathrm{r} 2 & =2.5 \mu \mathrm{m}\end{aligned}$ & $\begin{aligned} \mathrm{n} 1 & =1.83 \\
\mathrm{n} 2 & =1.60\end{aligned}$ & 7039 & 757 & 4120 & 2.52 & 23.4 \\
\hline \multirow{9}{*}{ TMLM } & & \multirow{3}{*}{$\begin{array}{c}\mathrm{n} 1=1.45, \\
\mathrm{n} 2=1.51, \mathrm{r} 1 \\
=2.4 \mu \mathrm{m} \\
\mathrm{r} 2=2.5 \mu \mathrm{m}\end{array}$} & $\begin{array}{c}\mathrm{T}=0.25 \mathrm{D} \\
= \\
1.25 \mu \mathrm{m}\end{array}$ & 9079 & 845 & 6466 & 2.80 & 30.1 \\
\hline & & & $\begin{array}{c}\mathrm{T}=0.50 \mathrm{D} \\
= \\
2.50 \mu \mathrm{m}\end{array}$ & 16179 & 1025 & 8476 & 2.42 & 38.2 \\
\hline & & & $\begin{array}{l}\mathrm{T}=0.75 \mathrm{D} \\
=3.75 \mu \mathrm{m}\end{array}$ & $77614 *$ & 1459 & 9629 & 1.33 & 70.8 \\
\hline & & \multirow{3}{*}{$\begin{array}{c}\mathrm{n} 1=1.51 \\
\mathrm{n} 2=1.38, \mathrm{r} 1 \\
=2.4 \mu \mathrm{m}, \mathrm{r} 2 \\
=2.5 \mu \mathrm{m}\end{array}$} & $\begin{array}{l}\mathrm{T}=0.25 \mathrm{D} \\
=1.25 \mu \mathrm{m}\end{array}$ & 69034 & 1938 & 19588 & 1.19 & 42.4 \\
\hline & & & $\begin{array}{l}\mathrm{T}=0.50 \mathrm{D} \\
=2.50 \mu \mathrm{m}\end{array}$ & 74754 & 2006 & 16617 & 1.13 & 42.1 \\
\hline & & & $\begin{array}{l}\mathrm{T}=0.75 \mathrm{D} \\
=3.75 \mu \mathrm{m}\end{array}$ & 137609 & 2977 & 25808 & 0.83 & 38.4 \\
\hline & & \multirow{3}{*}{$\begin{array}{c}\mathrm{n} 1=1.83, \\
\mathrm{n} 2=1.60, \mathrm{r} 1 \\
=2.0 \mu \mathrm{m}, \mathrm{r} 2 \\
=2.5 \mu \mathrm{m}\end{array}$} & $\begin{array}{l}\mathrm{T}=0.25 \mathrm{D} \\
=1.25 \mu \mathrm{m}\end{array}$ & 56036 & 949 & 4309 & 1.32 & 77.9 \\
\hline & & & $\begin{array}{l}\mathrm{T}=0.50 \mathrm{D} \\
=2.50 \mu \mathrm{m}\end{array}$ & 8099 & 831 & 6175 & 2.60 & 25.3 \\
\hline & & & $\begin{array}{l}\mathrm{T}=0.75 \mathrm{D} \\
=3.75 \mu \mathrm{m}\end{array}$ & 12399 & 869 & 6386 & 2.44 & 34.8 \\
\hline
\end{tabular}

Fig. 2 Properties of PNJ generated by microspheres (with diameter, $D=5 \mu \mathrm{m}$ ) of different designs when illuminated with a focusing Gaussian beam (generated in water with $0.1 \mathrm{NA}$ and $\lambda=800 \mathrm{~nm}$ ) in water medium ( $n 0=1.33)$. The FWHM waist diameter of Gaussian focus in water, $W_{g}=3958 \mathrm{~nm}$. The highlighted ones are the best values observed in our simulations. $N$-number of concentric rings. ${ }^{*}$ The PNJs used for demonstrating PA imaging in k-wave simulations, where $L_{p}$ is the effective length, $W_{p}$ is the FWHM width, WD is the working distance of the microsphere, $I_{p}$ is the peak intensity, and $Q$ is the quality criterion or figure-of-merit. 
optimized based on the $Q$ value of the nanojet they produced. The sphere inside the focusing Gaussian beam was moved (along $y$-axis) to generate a PNJ with highest $Q$ value. The PNJ parameters, such as the effective length $\left(L_{p}\right)$, FWHM waist size $\left(W_{p}\right)$, working distance (WD), and peak intensity $\left(I_{p}\right)$, and quality criterion $(Q)$ of the PNJs generated when a $5 \mu \mathrm{m}$ sphere was placed inside a focusing Gaussian beam with $3958 \mathrm{~nm}$ waist size, are listed in Fig. 2. The summary of the table is as follows: (i) The PNJ properties are significantly affected by the design of the sphere under the same illumination conditions. (ii) FWHM widths of all the PNJs $\left(W_{p}\right)$ are smaller than the Gaussian focus (i.e., $W_{g}=3958 \mathrm{~nm}$ ). (iii) Increase in $n_{1}$ will reduce the PNJ width, length, and working distance. The PNJ is elongated at the expense of PNJ broadening and intensity lowering. (iv) RM design could generate a strong and narrow nanojet. With $n_{1}=2.2$, PNJ having peak intensity $I_{p}=4.87$ and width $W_{p}=$ $360 \mathrm{~nm}$ was observed. The ultranarrrow nanojet having length $L_{p} \sim 1380 \mathrm{~nm}(\sim 1.73 \lambda)$ is shown in Fig. 3(a). The $n_{1}=2.2$ sphere generates a $360 \mathrm{~nm}$ spot from a $3958 \mathrm{~nm}$ Gaussian spot, i.e., $\sim 11$-flod enhancement in the resolution. But the drawback with the RM design is it could only generate a nanojet with short length and short working distance. (v) By cutting the silica
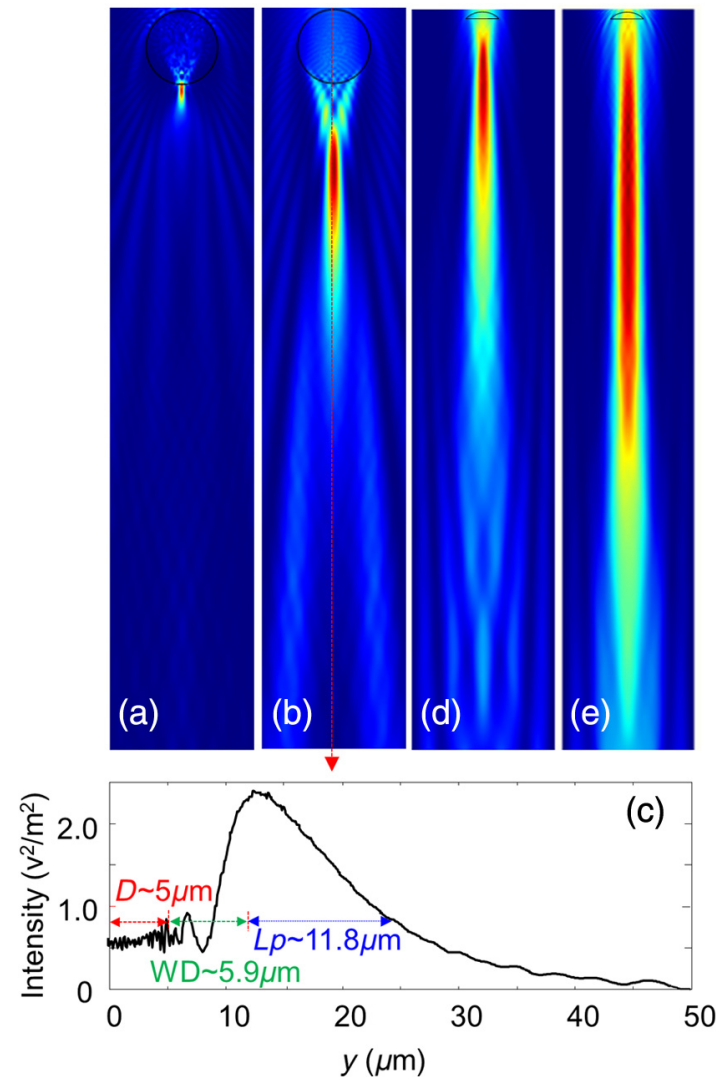

Fig. 3 Fluence maps generated when a Gaussian beam with waist $3958 \mathrm{~nm}$ focused through (a) an RM with $D=5 \mu \mathrm{m}$ and $n 1=2.2$, (b) an RM with $D=5 \mu \mathrm{m}$ and $n 1=1.45$, and (c) intensity profile along the line shown in (b). Here, $D$-diameter of the sphere $(5 \mu \mathrm{m})$, WD-working distance $(\sim 5.9 \mu \mathrm{m}), L_{p}$-effective length $(\sim 11.8 \mu \mathrm{m})$, (d) a TM with $D=5 \mu \mathrm{m}, n 1=1.45$, and $T=0.75 D=3.75 \mu \mathrm{m}$, and (e) a TMLM with $n 1=1.51$ and $n 2=1.38$. COMSOL simulations were carried out in $20 \mu \mathrm{m} \times 250 \mu \mathrm{m}$ area. For clarity, the area around the nanojet is shown here. The area of images in (a) and (b) is $10 \mu \mathrm{m} \times$ $50 \mu \mathrm{m}$ and the area of images in (c) and (d) is $20 \mu \mathrm{m} \times 170 \mu \mathrm{m}$. $n_{1}=1.45$, sphere by $0.75 D$, i.e., $3.75 \mu \mathrm{m}$, the PNJ was elongated from 11,779 to $74,314 \mathrm{~nm}$ (i.e., $\sim 6.3$-fold enhancement in length). Figure 3(b) shows the PNJ with the length of $11,779 \mathrm{~nm}$ obtained by round silica sphere and its intensity plot along the line indicated as shown in Fig. 3(c). Figure 3(d) shows the PNJ with the length of $74,314 \mathrm{~nm}$ obtained by truncating the silica sphere by $T=0.75 D$. (vi) In case of MCR design, the number of rings 2,3 , or 4 made on the round silica sphere does not influence the PNJ parameters much. However, the length and working distance are slightly better compared to the RM silica sphere $\left(n_{1}=1.45\right)$. (vi) By properly selecting the sphere and layer materials, the MLM design is promising to generate an ultralong nanojet. Here, the layer thickness was optimized to generate PNJ with highest $Q$ value. With $n_{1}=1.51, n 2=1.38$, $r 1=2.4 \mu \mathrm{m}, r 2=2.5 \mu \mathrm{m}$, a $47-\mu \mathrm{m}$ long nanojet was generated. (vii) The TMLM design with $n_{1}=1.51, n 2=1.38$, $r 1=2.4 \mu \mathrm{m}, r 2=2.5 \mu \mathrm{m}$, and cutting thickness $T=0.75 \mathrm{D}=$ $3.75 \mu$ m generated PNJ with a remarkable extension of $\sim 138 \mu \mathrm{m}$ $(\sim 172 \lambda$, it is the longest nanojet compared to the previously reported nanojet lengths $\left.{ }^{30,31}\right)$ and long working distance $\sim 26 \mu \mathrm{m}(\sim 32 \lambda$, it is the longest working distance compared to the previously reported values ${ }^{28,36}$ ). The ultralong nanojet is shown in Fig. 3(e). The fluence maps for Gaussian focus, nanojets from RM design with silica sphere $\left(n_{1}=1.45\right)$, and TMLM design with $n_{1}=1.45, n 2=1.51$, and $T=0.75 D$ are shown in Figs. 4(a)-4(c), respectively. Figure 4(d) shows the normalized FWHM line profiles showing the widths and lengths. The fluence maps for Gaussian focus and PNJs as shown in Figs. 4(a)-4(c) were then used for illuminating the object [Fig. 1(b)] in k-wave simulations in MATLAB to generate PA B-scan image.

\subsection{Super-Resolution Photoacoustic B-Scan Imaging}

The numerical phantom described in Fig. 1(b) was sequentially scanned by the fluence maps shown in Figs. 4(a)-4(c). Figures 5(a)-5(c) show the 2-D B-scan PAM images of the target excited with the Gaussian focused beam with FWHM $3958 \mathrm{~nm}$ [Fig. 4(a)] and excited with PNJs with FWHM $909 \mathrm{~nm}$ [Fig. 4(b)] and $1459 \mathrm{~nm}$ [Fig. 4(c)], respectively. The images shown in Figs. 5(a)-5(c) were acquired using $375 \mathrm{MHz}$ UST. From the ORPAM image shown in Fig. 5(a), it is clear that the Gaussian beam illumination could provide information of all the targets at depth but it could not resolve the gap between the targets. It is because the Gaussian spot size $\left(W_{g}=3958 \mathrm{~nm}\right)$ is larger than the separation between the targets $1500 \mathrm{~nm}$. Figure 5(b) shows that the narrow PNJ (909 nm) generated by $\mathrm{RM}$ could resolve the gap $(1500 \mathrm{~nm})$ between the targets but it could not image the targets beyond $10-\mu \mathrm{m}$ depth. From Fig. 5(c), it is clear that the elongated PNJ $(\sim 77,614 \mu \mathrm{m})$ generated using TMLM design could image all the targets and resolve the gap $(1500 \mathrm{~nm})$ between the targets. Here, the gap between the targets is clearly resolved, but the gap is not uniform along the depth. This is due to slight variation in the width along the length of the nanojet. Similar simulations were repeated using $1225 \mathrm{MHz}$ transducer and the corresponding ORPAM images are shown in Figs. 5(d)-5(f). From these results, it is evident that the depth of super-resolution ORPAM can be enhanced with the help of ultralong PNJs.

These simulations promise that it is possible to control or tune the imaging depth, lateral resolution, and signal enhancement in the conventional ORPAM system just by changing the sphere design being used. Based on the requirement, the PNJ 
FGB, $W g=3958 \mathrm{~nm}$ RM, $W p=909 \mathrm{~nm}$ TMLM, $W p=1459 \mathrm{~nm}$
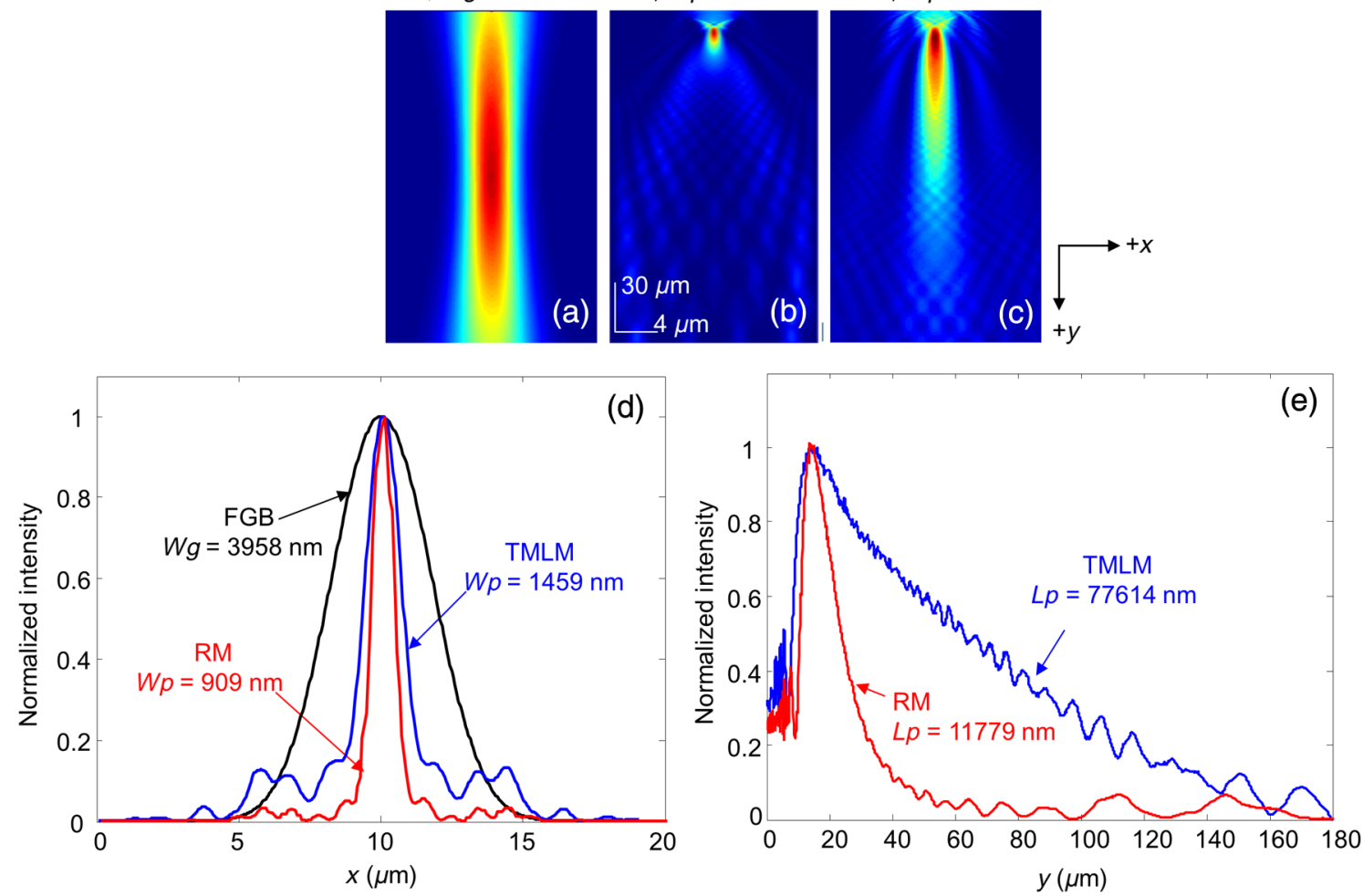

Fig. 4 Fluence maps for Gaussian focus and PNJs: (a) focusing Gaussian beam in water with $\lambda=800 \mathrm{~nm}, \mathrm{NA}=0.1$. PNJs generated when the Gaussian beam (a) focused on (b) round sphere with $D=5 \mu \mathrm{m}$ and $n 1=1.45$, (c) TMLM with $D=5 \mu \mathrm{m}, n 1=1.45$, and $n 2=1.51$. Normalized line profiles showing (d) widths and (e) lengths. The area of images in (a)-(c) is $20 \mu \mathrm{m} \times 180 \mu \mathrm{m}$.
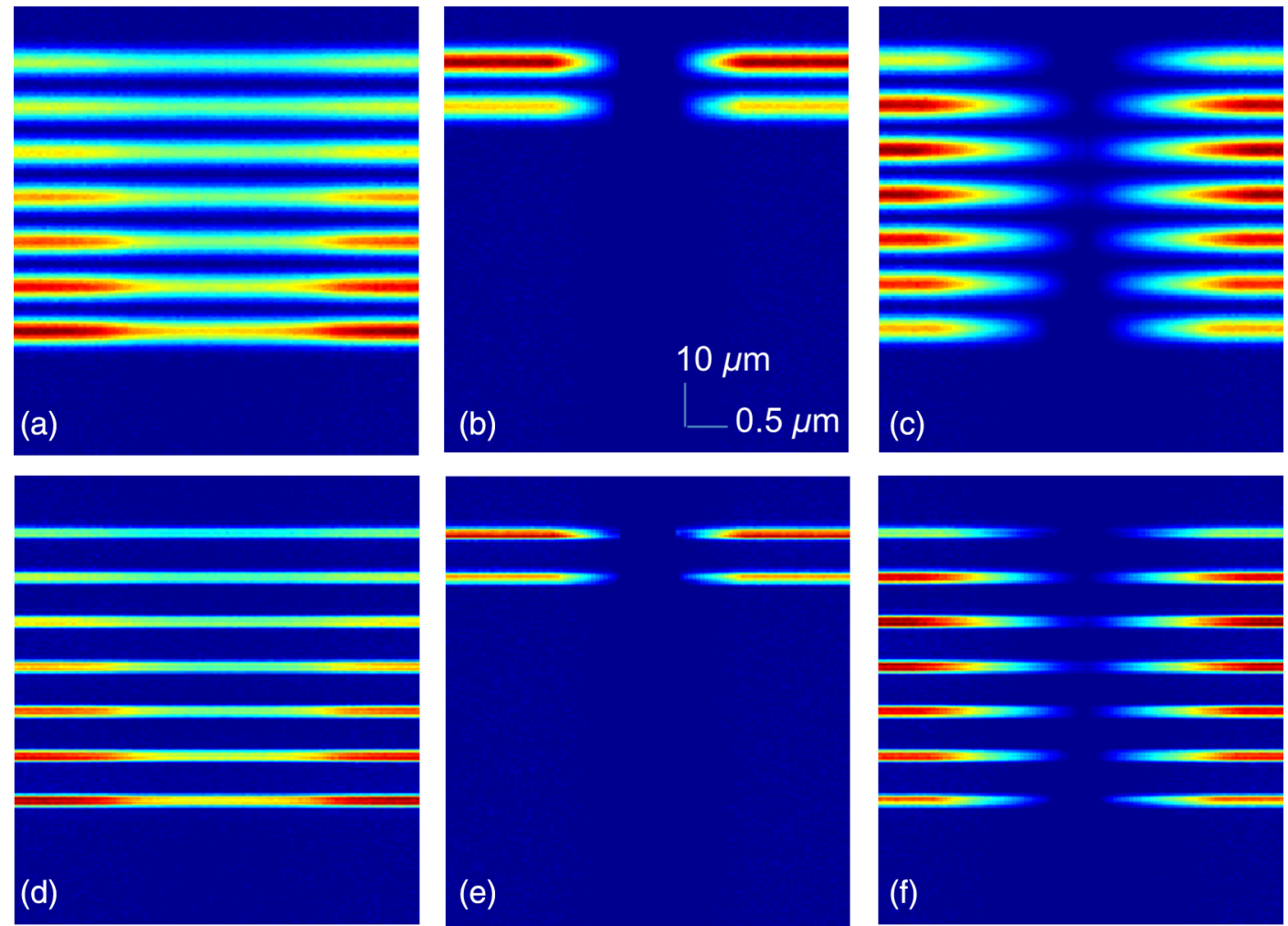

(d)

Fig. 5 PAM B-scan images obtained by scanning the object [Fig. 1(b)] with Gaussian focus and PNJ: (a, d) images obtained with Gaussian focus shown in Fig. 4(a), (b, e) images obtained with short $(\sim 11,779 \mathrm{~nm})$ PNJ shown in Fig. 4(b), and (c, f) images obtained with ultralong ( 77,614 nm) PNJ shown in Fig. 4(c). Images were acquired using USTs (a-c) $375 \mathrm{MHz}$ and (d-f) $1225 \mathrm{MHz}$. 
parameters can be optimized by choosing the appropriate sphere and refractive index. Microsphere has already proven its potential in white light microscopy ${ }^{21}$ by imaging 50 -nm features, and in fluorescence microscopy ${ }^{24}$ by imaging subcellular structures such as centrioles, mitochondria, and chromosomes, and also in Raman microscopy by imaging subdiffraction features of a Bluray disc. ${ }^{26}$ The simulation results in our work promise that by using an appropriate microsphere, conventional ORPAM could be transformed into PAN at virtually no extra cost. The PAN can be used in many biomedical imaging applications that demand high resolution. For example, cellular structures, vasculature, and microcirculation systems, in vivo PAN, having high endogenous contrast, may help to monitor the molecular mechanism of blood vessel formation that can reveal the cause for angiogenesis. Currently, the diagnosis of melanoma is done based on invasive biopsy and inaccurate visual inspection. In vivo PAN being noninvasive, noncontact, and label-free will help us to diagnose melanoma in the early stage, a key to successful treatment. The approach may allow ORPA imaging of biological entities, such as bacteria $(\sim 1 \mu \mathrm{m})$, large virus (0.05 to $0.4 \mu \mathrm{m})$ particles, labeled as nucleic acids, molecules, etc. In spite of its promises, there are challenges in implementing the microsphere approach in practice. Although, other optical imaging modalities have successfully demonstrated the use of PNJ to improve the imaging resolution by breaking the diffraction limit. The experimental validation of PNJ for PA imaging is yet to be demonstrated. Our future work is to experimentally validate the usefulness of this simple technique in optical-resolution PA imaging.

\section{Conclusions}

In this work, we investigated and optimized the main parameters of PNJ (length, width, working distance, and peak intensity) for various spheres under Gaussian illumination in water medium. The materials used for spheres are conventionally available in optical materials. The RM generates strong, short, and narrow nanojets, it can be used for super-resolution imaging of surface and nano/submicron features on the surface. With $5 \mu \mathrm{m} \mathrm{RM}$ having $n 1=2.2$, an ultranarrow nanojet having 360-nm width ( 11-fold enhancement in lateral resolution) was observed. An elongated nanojet can be generated by simply truncating the microsphere by an appropriate thickness. The TMLM could generate PNJ with remarkable length $\sim 138 \mu \mathrm{m}(\sim 172 \lambda)$ and working distance $\sim 26 \mu \mathrm{m}(\sim 32 \lambda)$. Also, the TMLM design could generate PNJ with highest figure-of-merit, $Q \sim 78$, compared to the other designs. The method is simple, cost-effective, and allows far-field super-resolution in vivo imaging.

\section{Disclosures}

No conflicts of interest, financial or otherwise, are declared by the authors.

\section{Acknowledgments}

The authors would like to acknowledge the financial support from the Tier 2 research grant funded by the Ministry of Education in Singapore (ARC2/15: M4020238). Authors have no relevant financial interests in the manuscript and no other potential conflicts of interest to disclose.

\section{References}

1. S.-L. Chen, L. J. Guo, and X. Wang, "All-optical photoacoustic microscopy," Photoacoustics 3(4), 143-150 (2015).

2. L. V. Wang and S. Hu, "Photoacoustic tomography: in vivo imaging from organelles to organs," Science 335(6075), 1458-1462 (2012).

3. S. S. Oladipupo et al., "Conditional HIF-1 induction produces multistage neovascularization with stage-specific sensitivity to VEGFR inhibitors and myeloid cell independence," Blood 117(15), 4142-4153 (2011).

4. X. Cai et al., "Photoacoustic tomography of foreign bodies in soft biological tissue," J. Biomed. Opt. 16(4), 046017 (2011).

5. D. Pan et al., "Molecular photoacoustic imaging of angiogenesis with integrin-targeted gold nanobeacons," FASEB J. 25(3), 875-882 (2011).

6. S. Hu et al., "Label-free photoacoustic ophthalmic angiography," Opt. Lett. 35(1), 1-3 (2010).

7. M. Schwarz et al., "Three-dimensional multispectral optoacoustic mesoscopy reveals melanin and blood oxygenation in human skin in vivo," J. Biophotonics 9(1-2), 55-60 (2016).

8. H. F. Zhang et al., "Functional photoacoustic microscopy for highresolution and noninvasive in vivo imaging," Nat. Biotechnol. 24(7), 848-851 (2006).

9. J. Yao et al., "Photoimprint photoacoustic microscopy for three-dimensional label-free subdiffraction imaging," Phys. Rev. Lett. 112(1), 014302 (2014).

10. G. Li, K. I. Maslov, and L. V. Wang, "Reflection-mode multifocal optical-resolution photoacoustic microscopy," J. Biomed. Opt. 18(3), 030501 (2013).

11. W. Shi et al., "In vivo dynamic process imaging using real-time opticalresolution photoacoustic microscopy," J. Biomed. Opt. 18(2), 026001 (2013).

12. C. Zhang, K. Maslov, and L. V. Wang, "Subwavelength-resolution label-free photoacoustic microscopy of optical absorption in vivo," Opt. Lett. 35(19), 3195-3197 (2010).

13. K. Maslov et al., "Optical-resolution photoacoustic microscopy for in vivo imaging of single capillaries," Opt. Lett. 33(9), 929-931 (2008).

14. P. Hai et al., "Near-infrared optical-resolution photoacoustic microscopy," Opt. Lett. 39(17), 5192-5195 (2014).

15. G. $\mathrm{Ku}$ et al., "Photoacoustic microscopy with 2-microm transverse resolution," J. Biomed. Opt. 15(2), 021302 (2010).

16. D.-K. Yao et al., "In vivo label-free photoacoustic microscopy of cell nuclei by excitation of DNA and RNA," Opt. Lett. 35(24), 4139-4141 (2010).

17. D. A. Nedosekin et al., "Super-resolution nonlinear photothermal microscopy," Small 10(1), 135-142 (2014).

18. A. Danielli et al., "Label-free photoacoustic nanoscopy," J. Biomed. Opt. 19(8), 086006 (2014).

19. S. Lee et al., "Overcoming the diffraction limit induced by microsphere optical nanoscopy,” J. Opt. 15(12), 125710 (2013).

20. A. Darafsheh et al., "Optical super-resolution by high-index liquid-immersed microspheres," Appl. Phys. Lett. 101(14), 141128 (2012).

21. Z. Wang et al., "Optical virtual imaging at $50 \mathrm{~nm}$ lateral resolution with a white-light nanoscope," Nat. Commun. 2, 218 (2011).

22. A. Heifetz et al., "Photonic nanojets," J. Comput. Theor. Nanosci. 6(9), 1979-1992 (2009).

23. H. Yang et al., "Super-resolution imaging of a dielectric microsphere is governed by the waist of its photonic nanojet," Nano Lett. 16(8), 4862-4870 (2016).

24. H. Yang et al., "Super-resolution biological microscopy using virtual imaging by a microsphere nanoscope," Small 10(9), 1712-1718 (2014).

25. J. Kasim et al., "Near-field Raman imaging using optically trapped dielectric microsphere," Opt. Express 16(11), 7976-7984 (2008).

26. P. K. Upputuri et al., "Super-resolution coherent anti-Stokes Raman scattering microscopy with photonic nanojets," Opt. Express 22(11), 12890-12899 (2014).

27. P. K. Upputuri et al., "Super-resolution photoacoustic microscopy using photonic nanojets: a simulation study," J. Biomed. Opt. 19(11), 116003 (2014).

28. C.-Y. Liu, "Photonic nanojet shaping of dielectric non-spherical microparticles," Phys. E 64, 23-28 (2014). 
29. M. X. Wu et al., "Modulation of photonic nanojets generated by microspheres decorated with concentric rings," Opt. Express 23(15), 20096 (2015).

30. G. Gu et al., "Super-long photonic nanojet generated from liquid-filled hollow microcylinder," Opt. Lett. 40(4), 625-628 (2015).

31. Y. Shen, L. V. Wang, and J. T. Shen, "Ultralong photonic nanojet formed by a two-layer dielectric microsphere," Opt. Lett. 39(14), 4120-4123 (2014).

32. B. E. Treeby and B. T. Cox, "k-wave: MATLAB toolbox for the simulation and reconstruction of photoacoustic wave fields," J. Biomed. Opt. 15(2), 021314 (2010).

33. E. M. Strohm, M. J. Moore, and M. C. Kolios, "High resolution ultrasound and photoacoustic imaging of single cells," Photoacoustics 4(1), 36-42 (2016).

34. E. M. Strohm, M. J. Moore, and M. C. Kolios, "Single cell photoacoustic microscopy: a review," IEEE J. Sel. Top. Quantum Electron. 22(3), 6801215 (2016).

35. E. M. Strohm, E. S. Berndl, and M. C. Kolios, "High frequency labelfree photoacoustic microscopy of single cells," Photoacoustics 1(3-4), 49-53 (2013).

36. P. Wu et al., "Tunable and ultra-elongated photonic nanojet generated by a liquid-immersed core-shell dielectric microsphere," Appl. Phys. Express 8(11), 112001 (2015).
Paul Kumar Upputuri received his $\mathrm{PhD}$ in physics from the Indian Institute of Technology (IIT), Madras, in 2010. Currently, he is working as a research fellow at the Nanyang Technological University. He has published around 65 papers in journals and conference proceedings. His research areas are biomedical imaging, coherent Raman microscopy, ultrafast lasers, optical metrology, and instrumentation. He is a member of SPIE, OSA, Indian Laser Association (ILA), and Optical Society of India (OSI).

Moganasundari Krisnan received her bachelor's degree in biomedical engineering from the Nanyang Technological University, Singapore, in 2016.

Manojit Pramanik received his $\mathrm{PhD}$ in biomedical engineering from Washington University in St. Louis, Missouri, USA. Currently, he is an assistant professor at the School of Chemical and Biomedical Engineering, Nanyang Technological University, Singapore. His research interests include the development of photoacoustic/ thermoacoustic imaging systems, image reconstruction methods, and clinical application areas such as breast cancer imaging, molecular imaging, contrast agent development, and Monte Carlo simulation of light propagation in biological tissue. 